PROCEEDINGS OF THE

AMERICAN MATHEMATICAL SOCIETY

Volume 138, Number 6, June 2010, Pages 1923-1927

S 0002-9939(10)10205-6

Article electronically published on February 5, 2010

\title{
ON ADDITIVE COMPLEMENTS
}

\author{
JIN-HUI FANG AND YONG-GAO CHEN
}

(Communicated by Wen-Ching Winnie Li)

\begin{abstract}
Two infinite sequences $A$ and $B$ of non-negative integers are called additive complements if their sum contains all sufficiently large integers. Let $A(x)$ and $B(x)$ be the counting functions of $A$ and $B$. For additive complements $A$ and $B$, Sárközy and Szemerédi proved that if $\limsup \frac{A(x) B(x)}{x} \leq 1$, then $A(x) B(x)-x \rightarrow+\infty$. In this paper, we prove that for additive complements $A$ and $B$, if $\limsup \frac{A(x) B(x)}{x}<\frac{5}{4}$ or $\limsup \frac{A(x) B(x)}{x}>2$, then $A(x) B(x)-x \rightarrow+\infty$.
\end{abstract}

\section{INTRODUCTION}

Two infinite sequences $A$ and $B$ of non-negative integers are called additive complements if their sum contains all sufficiently large integers. Let $A(x)$ and $B(x)$ be the counting functions of $A$ and $B$, namely,

$$
A(x)=\sum_{\substack{a \leq x \\ a \in A}} 1 \text { and } B(x)=\sum_{\substack{b \leq x \\ b \in B}} 1 .
$$

Motivated by a problem of Hanani and Erdös 2, [3, Danzer [1] conjectured that for additive complements $A$ and $B$, if

$$
\limsup _{x \rightarrow \infty} \frac{A(x) B(x)}{x} \leq 1
$$

then

$$
A(x) B(x)-x \rightarrow+\infty \quad \text { as } \quad x \rightarrow+\infty .
$$

(See also [4, p. 10], [5, p. 75] and [6].) In [8, Sárközy and Szemerédi proved this conjecture.

In this paper, we prove the following result.

Theorem. For additive complements $A$ and $B$, if

$$
\limsup _{x \rightarrow \infty} \frac{A(x) B(x)}{x}>2
$$

Received by the editors July 1, 2009, and, in revised form, September 10, 2009

2010 Mathematics Subject Classification. Primary 11B13, 11 B34.

Key words and phrases. Additive complements, sequences, counting functions.

This work was supported by the National Natural Science Foundation of China, Grant No. 10771103 and the Outstanding Graduate Dissertation Program of Nanjing Normal University, Grant No. 181200000213.

(C) 2010 American Mathematical Society
Reverts to public domain 28 years from publication 
or

then (1) must hold.

$$
\limsup _{x \rightarrow \infty} \frac{A(x) B(x)}{x}<\frac{5}{4}
$$

For the construction of additive complements $A$ and $B$ with $A(x) B(x) \sim x$ one may refer to [1] and [7].

\section{Proof of the Theorem}

Let $f(n)$ be the number of solutions of $a+b=n$, for $a \in A, b \in B$. For additive complements $A$ and $B$, there exists a constant $n_{0}$ such that

$$
f(n) \geq 1 \text { for } n>n_{0} \text {. }
$$

Hence $A(x) B(x) \geq[x]-n_{0}$.

If (1) does not hold, then $-\infty<\liminf _{x \rightarrow \infty}(A(x) B(x)-x)<+\infty$. Assume that

$$
\liminf _{x \rightarrow \infty}(A(x) B(x)-x)=L .
$$

By the same arguments as in Sárközy and Szemerédi [8, p. 238], there exists an integer $n_{1}$ with

$$
f(n) \equiv 1 \quad \text { for } n \geq n_{1}
$$

Then

$$
A(x) B(x) \leq \sum_{n \leq 2 x} f(n) \leq \sum_{n \leq n_{1}} f(n)+\left(2 x-n_{1}\right)=2 x+O(1)
$$

Hence

$$
\limsup _{x \rightarrow \infty} \frac{A(x) B(x)}{x} \leq 2
$$

Thus, if

$$
\limsup _{x \rightarrow \infty} \frac{A(x) B(x)}{x}>2
$$

then

$$
\liminf _{x \rightarrow \infty}(A(x) B(x)-x)=+\infty
$$

Now we assume that the additive complements $A$ and $B$ satisfy

$$
\limsup _{x \rightarrow \infty} \frac{A(x) B(x)}{x}=M \text {. }
$$

Then $A(x) B(x) \leq x(M+o(1))$. Since $A$ and $B$ are infinite, we have $A(x)=o(x)$ and $B(x)=o(x)$.

Let $x_{1}<x_{2}<\cdots$ be all positive integers with $A\left(x_{k}\right) B\left(x_{k}\right)-x_{k}=L, b^{(k)}$ be the largest integer in $(A \cup B) \cap\left[0, x_{k}\right]$ and $y_{k}=x_{k}-b^{(k)}$. By the same discussion as in [8], we know that $y_{k} \rightarrow+\infty$. Since

$$
\begin{aligned}
x_{k}+L=A\left(x_{k}\right) B\left(x_{k}\right) & =A\left(b^{(k)}\right) B\left(b^{(k)}\right) \\
& =A\left(x_{k}-y_{k}\right) B\left(x_{k}-y_{k}\right) \leq\left(M+o_{k}(1)\right)\left(x_{k}-y_{k}\right),
\end{aligned}
$$

we have

$$
y_{k} \leq\left(1-\frac{1}{M}\right) x_{k}+o_{k}\left(x_{k}\right)
$$

As in 8] we know that

$$
A\left(x_{k}\right)-A\left(y_{k}\right) \leq L+n_{0}
$$


and

$$
A\left(x_{k}\right)-A\left(2 y_{k}\right)=0, \quad k \geq k_{0} .
$$

Define $D, D_{1}, D_{1}^{+}$and $D_{1}^{-}$in the same way as in $[8$, namely,

$$
\begin{gathered}
D=\left\{(b, a): b \in B, a \in A, b \leq x_{k}-y_{k}, a \leq x_{k}-y_{k}, b-a>y_{k}\right\}, \\
D_{1}=\left\{(b, a): b \in B, a \in A, 2 y_{k}<b \leq x_{k}-y_{k}, b-a>y_{k}\right\}, \\
D_{1}^{+}=\left\{(b, a): b \in B, a \in A, 2 y_{k}<b \leq x_{k}-y_{k}, a \leq 2 y_{k}\right\}, \\
D_{1}^{-}=\left\{(b, a): b \in B, a \in A, 2 y_{k}<b \leq x_{k}-y_{k}, a \leq 2 y_{k}, a \geq b-y_{k}\right\} .
\end{gathered}
$$

By $A\left(x_{k}\right)=A\left(2 y_{k}\right)$ for $k \geq k_{0}$, we have $\left|D_{1}\right|=\left|D_{1}^{+}\right|-\left|D_{1}^{-}\right|$. As in [8], it suffices to show that for large $k,|D|>x_{k}-2 y_{k}$.

Fix an integer $t$ with

$$
t>\frac{1}{\frac{5}{4}-M}
$$

Let

$$
\alpha_{i}=1+\frac{i-1}{t} \quad(1 \leq i \leq t+1)
$$

and

$$
A\left(\alpha_{i} y_{k}\right) B\left(\alpha_{i} y_{k}\right)=M_{i} \alpha_{i} y_{k}
$$

Obviously, $1-o_{t, i}(1) \leq M_{i} \leq M+o_{t, i}(1)$. For $D_{1}^{+}$we have

$$
\begin{aligned}
\left|D_{1}^{+}\right| & =\left(B\left(x_{k}-y_{k}\right)-B\left(2 y_{k}\right)\right) A\left(2 y_{k}\right)=B\left(b^{(k)}\right) A\left(2 y_{k}\right)-B\left(2 y_{k}\right) A\left(2 y_{k}\right) \\
& =B\left(x_{k}\right) A\left(x_{k}\right)-B\left(2 y_{k}\right) A\left(2 y_{k}\right)=\left(x_{k}+O_{k}(1)\right)-2 M_{t+1} y_{k} .
\end{aligned}
$$

For $D_{1}^{-}$, as in 8 , we have $\left|D_{1}^{-}\right|=o\left(y_{k}\right)$. Hence

$$
\left|D_{1}\right|=x_{k}-2 M_{t+1} y_{k}+o\left(y_{k}\right) .
$$

We redefine $D_{2}$ as

$$
D_{2}=\left\{(b, a): b \in B, a \in A, b \leq 2 y_{k}, b-a>y_{k}\right\} .
$$

Obviously, $D_{1} \cap D_{2}=\emptyset$ and $D=D_{1} \cup D_{2}$. Hence $|D|=\left|D_{1}\right|+\left|D_{2}\right|$.

Now we are going to estimate $\left|D_{2}\right|$. It follows from the definition of $D_{2}$ that

$$
\begin{aligned}
\left|D_{2}\right| & =\sum_{y_{k}<b \leq 2 y_{k}} A\left(b-y_{k}\right)=\sum_{i=1}^{t} \sum_{\alpha_{i} y_{k}<b \leq \alpha_{i+1} y_{k}} A\left(b-y_{k}\right) \\
& \geq \sum_{i=1}^{t} A\left(\left(\alpha_{i}-1\right) y_{k}\right)\left(B\left(\alpha_{i+1} y_{k}\right)-B\left(\alpha_{i} y_{k}\right)\right) .
\end{aligned}
$$

Since

$$
A\left(\left(\alpha_{i}-1\right) y_{k}\right) \geq \frac{A\left(\left(\alpha_{i}-1\right) y_{k}\right) B\left(\left(\alpha_{i}-1\right) y_{k}\right)}{A\left(y_{k}\right) B\left(y_{k}\right)} A\left(y_{k}\right) \geq\left(\frac{\alpha_{i}-1}{M}-o_{k}(1)\right) A\left(y_{k}\right)
$$

and

$$
\begin{aligned}
& B\left(\alpha_{i+1} y_{k}\right)-B\left(\alpha_{i} y_{k}\right)=\frac{B\left(\alpha_{i+1} y_{k}\right) A\left(y_{k}\right)-B\left(\alpha_{i} y_{k}\right) A\left(y_{k}\right)}{A\left(y_{k}\right)} \\
= & \frac{B\left(\alpha_{i+1} y_{k}\right)\left(A\left(\alpha_{i+1} y_{k}\right)+O_{k}(1)\right)-B\left(\alpha_{i} y_{k}\right)\left(A\left(\alpha_{i} y_{k}\right)+O_{k}(1)\right)}{A\left(y_{k}\right)} \\
= & \left(M_{i+1} \alpha_{i+1}-M_{i} \alpha_{i}+o_{k}(1)\right) \frac{y_{k}}{A\left(y_{k}\right)},
\end{aligned}
$$


we have

$$
\begin{aligned}
\left|D_{2}\right| & \geq \sum_{i=1}^{t}\left(\frac{\alpha_{i}-1}{M}\left(M_{i+1} \alpha_{i+1}-M_{i} \alpha_{i}\right)\right) y_{k}+o_{t}\left(y_{k}\right) \\
& =\frac{1}{M}\left(-\sum_{i=2}^{t+1} M_{i} \alpha_{i}\left(\alpha_{i}-\alpha_{i-1}\right)+M_{t+1} \alpha_{t+1}\left(\alpha_{t+1}-1\right)\right) y_{k}+o_{t}\left(y_{k}\right) \\
& \geq \frac{1}{M}\left(-\left(M+o_{t}(1)\right) \sum_{i=2}^{t+1} \alpha_{i}\left(\alpha_{i}-\alpha_{i-1}\right)+2 M_{t+1}\right) y_{k}+o_{t}\left(y_{k}\right) \\
& =\frac{1}{M}\left(-\frac{3}{2} M+2 M_{t+1}-\frac{M}{2 t}\right) y_{k}+o_{t}\left(y_{k}\right) .
\end{aligned}
$$

Thus by $t>\frac{1}{\frac{5}{4}-M}$ we have

$$
\begin{aligned}
|D| & \geq x_{k}+\left(-2 M_{t+1}-\frac{3}{2}+\frac{2 M_{t+1}}{M}-\frac{1}{2 t}\right) y_{k}+o_{t}\left(y_{k}\right) \\
& \geq x_{k}+\left(-2 M_{t+1}\left(1-\frac{1}{M}\right)-\frac{3}{2}-\frac{1}{2 t}\right) y_{k}+o_{t}\left(y_{k}\right) \\
& \geq x_{k}+\left(-2\left(M+o_{t}(1)\right)\left(1-\frac{1}{M}\right)-\frac{3}{2}-\frac{1}{2 t}\right) y_{k}+o_{t}\left(y_{k}\right) \\
& \geq x_{k}+\left(-2 M+\frac{1}{2}-\frac{1}{2 t}\right) y_{k}+o_{t}\left(y_{k}\right)>x_{k}-2 y_{k}, \quad k>k_{1} .
\end{aligned}
$$

By the same discussion as in [ $[$, we obtain a contradiction, which completes the proof of the theorem.

\section{ACKNOWLEDGEMENT}

We would like to thank the referee for his/her kind comments.

\section{REFERENCES}

[1] L. Danzer, Über eine Frage von G. Hanani aus der additiven Zahlentheorie, J. Reine Angew. Math. 214/215(1964), 392-394. MR0161830 (28:5034)

[2] P. Erdős, Some unsolved problems, Mich. Math. J. 4(1957), 291-300. MR0098702 (20:5157)

[3] P. Erdős, Some unsolved problems, Publ. Math. Inst. Hung. Acad. Sci. Ser. A 6(1961), 221-254. MR0177846 (31:2106)

[4] P. Erdős, R. L. Graham, Old and New Problems and Results in Combinatorial Number Theory, Monographies de L'Enseignement Mathématique, 28, Université de Genève (1980). MR592420 (82j:10001)

[5] H. Halberstam, K. F. Roth, Sequences, 2nd ed., Springer-Verlag, New York-Berlin (1983). MR687978 (83m:10094)

[6] W. Narkiewicz, Remarks on a conjecture of Hanani in additive number theory, Colloq. Math. 7(1959/60), 161-165. MR0112876(22:3722) 
[7] I. Z. Ruzsa, Additive completion of lacunary sequences, Combinatorica 21(2)(2001), 279-291. MR.1832452 (2002f:11008)

[8] A. Sárközy, E. Szemerédi, On a problem in additive number theory, Acta Math. Hungar. 64(3)(1994), 237-245. MR1275641(95c:11121)

School of Mathematical Sciences, Nanjing Normal University, Nanjing 210046, PeoPLE'S REPUBLIC OF CHINA

E-mail address: fangjinhui1114@163.com

School of Mathematical Sciences, Nanjing Normal University, Nanjing 210046, PeoPLE'S REPUBlic OF CHINA

E-mail address: ygchen@njnu.edu.cn 